\title{
Parametrizing the Neutrino sector of the seesaw extension in tau decays
}

\section{Darius Jurciukonis ${ }^{*}$, , Thomas Gajdosik ${ }^{b}$, Andrius Juodagalvis ${ }^{a}$ and Tomas Sabonis $^{a}$}

${ }^{a}$ Vilnius University, Institute of Theoretical Physics and Astronomy

${ }^{b}$ Vilnius University, Physics Faculty

E-mail: Darius.Jurciukonis@cern.ch, Thomas.Gajdosik@cern.ch, Andrius. Juodagalvis@cern.ch, Tomas.Sabonisetfai.vu.lt

\begin{abstract}
The Standard Model includes neutrinos as massless particles, but neutrino oscillations showed, that neutrinos are not massless. A simple extension of adding gauge singlet fermions to the particle spectrum allows normal Yukawa mass terms for neutrinos. The smallness of the neutrino masses can be well understood within the seesaw mechanism. We analyse two cases of the minimal extension of the standard model when are added one or two right-handed fields to the three left-handed fields. In this model second Higgs doublet is included. We calculate the one-loop radiative corrections to the mass parameters which produce mass terms for the neutral leptons. In both cases we numerically analyse light neutrino masses as functions of the heavy neutrinos masses. Parameters of the model are varied to find light neutrino masses that are compatible with experimental data of solar $\Delta m_{\odot}^{2}$ and atmospheric $\Delta m_{\text {atm }}^{2}$ neutrino oscillations for normal and inverted hierarchy.
\end{abstract}

36th International Conference on High Energy Physics

4-11 July 2012

Melbourne, Australia

\footnotetext{
* Speaker.
} 


\section{The model}

We discus the extension of the Standard Model (SM) with a second Higgs doublet $\Phi_{\alpha}, \alpha=$ 1,2 with added right-handed neutrino fields to the three left handed neutrino fields. The Yukawa Lagrangian of the leptons is expressed by

$$
\mathscr{L}_{\mathrm{Y}}=-\sum_{k=1}^{2}\left(\Phi_{k}^{\dagger} \bar{\ell}_{R} \Gamma_{k}+\tilde{\Phi}_{k}^{\dagger} \bar{v}_{R} \Delta_{k}\right) D_{L}+\text { H.c. }
$$

in a vector and matrix notation, where $\tilde{\Phi}_{k}=i \tau_{2} \Phi_{k}^{*}$. In expression (1.1) the $\ell_{R}, v_{R}$, and $D_{L}=\left(v_{L} \ell_{L}\right)^{T}$ are the vectors of the right-handed charged leptons, of the right-handed neutrino singlets, and of the left-handed lepton doublets, respectively. The Yukawa coupling matrices $\Gamma_{k}$ are $n_{L} \times n_{L}$, while the $\Delta_{k}$ are $n_{R} \times n_{L}$.

In this model, spontaneous symmetry breaking of the SM gauge group is achieved by the vacuum expectation values $\left\langle\Phi_{k}\right\rangle_{\text {vak }}=\left(\begin{array}{c}0 \\ v_{k} / \sqrt{2}\end{array}\right)$. By a unitary rotation of the Higgs doublets, we can achieve $\left\langle\Phi_{1}^{0}\right\rangle_{\text {vak }}=v / \sqrt{2}>0$ and $\left\langle\Phi_{2}^{0}\right\rangle_{\text {vak }}=0$ with $v \simeq 246 \mathrm{GeV}$. The charged-lepton mass matrix $M_{\ell}$ and the Dirac neutrino mass matrix $M_{D}$ are

$$
M_{\ell}=\frac{v}{\sqrt{2}} \Gamma_{1} \quad \text { and } \quad M_{D}=\frac{v}{\sqrt{2}} \Delta_{1}
$$

respectively with assumption that $M_{\ell}=\operatorname{diag}\left(m_{e}, m_{\mu}, m_{\tau}\right)$. The mass terms for the neutrinos can be written in a compact form with an $\left(n_{L}+n_{R}\right) \times\left(n_{L}+n_{R}\right)$ symmetric mass matrix

$$
M_{v}=\left(\begin{array}{cc}
0 & M_{D}^{T} \\
M_{D} & \hat{M}_{R}
\end{array}\right)
$$

where the hat indicates that $\hat{M}_{R}$ is a diagonal matrix. $M_{v}$ can be diagonalized as

$$
U^{T} M_{v} U=\hat{m}=\operatorname{diag}\left(m_{1}, m_{2}, \ldots, m_{n_{L}+n_{R}}\right),
$$

where the $m_{i}$ are real and non-negative. In order to implement the seesaw mechanism $[1,2]$ we assume that the elements of $M_{D}$ are of order $m_{D}$ and those of $M_{R}$ are of order $m_{R}$, with $m_{D} \ll m_{R}$. Then, the neutrino masses $m_{i}$ with $i=1,2, \ldots, n_{L}$ are of order $m_{D}^{2} / m_{R}$, while those with $i=n_{L}+$ $1, \ldots, n_{L}+n_{R}$ are of order $m_{R}$. It is useful to decompose the $\left(n_{L}+n_{R}\right) \times\left(n_{L}+n_{R}\right)$ unitary matrix $U$ as $U=\left(\begin{array}{c}U_{L} \\ U_{R}^{*}\end{array}\right)$, where the submatrix $U_{L}$ is $n_{L} \times\left(n_{L}+n_{R}\right)$ and the submatrix $U_{R}$ is $n_{R} \times\left(n_{L}+n_{R}\right)[3,4]$. With these submatrices, the left- and right-handed neutrinos are written as linear superpositions of the $n_{L}+n_{R}$ physical Majorana neutrino fields $\chi_{i}: v_{L}=U_{L} P_{L} \chi$ and $v_{R}=U_{R} P_{R} \chi$, where $P_{L}$ and $P_{R}$ are the projectors of chirality.

We can express the couplings of the model in terms of mass eigenfields, where three neutral particles are coupling to neutrinos. The interaction of the $Z$ boson with the neutrinos is given by

$$
\mathscr{L}_{\mathrm{nc}}^{(v)}=\frac{g}{4 c_{w}} Z_{\mu} \bar{\chi} \gamma^{\mu}\left[P_{L}\left(U_{L}^{\dagger} U_{L}\right)-P_{R}\left(U_{L}^{T} U_{L}^{*}\right)\right] \chi,
$$

where $g$ is the $\mathrm{SU}(2)$ gauge coupling constant and $c_{w}$ is the cosine of the Weinberg angle. 
Full formalism for the scalar sector of the multi-Higgs-doublet SM is given in Ref. [3, 4]. The Yukawa couplings of the Higgs bosons $H_{b}^{0}$ to the neutrinos are given by

$$
\mathscr{L}_{\mathrm{Y}}^{(v)}\left(H^{0}\right)=-\frac{1}{2 \sqrt{2}} \sum_{b} H_{b}^{0} \bar{\chi}\left[\left(U_{R}^{\dagger} \Delta_{b} U_{L}+U_{L}^{T} \Delta_{b}^{T} U_{R}^{*}\right) P_{L}+\left(U_{L}^{\dagger} \Delta_{b}^{\dagger} U_{R}+U_{R}^{T} \Delta_{b}^{*} U_{L}^{*}\right) P_{R}\right] \chi,
$$

with $\Delta_{b}=\sum_{k} b_{k} \Delta_{k}$, where $b$ are two-dimensional complex unit vectors. The neutral Goldstone boson $G_{b_{Z}}^{0}$ is given by the vector $b_{Z}$ with $b_{Z}=(i, 0)$.

Once the one-loop corrections are taken into account the neutral fermion mass matrix is given by

$$
M_{v}^{(1)}=\left(\begin{array}{cc}
\delta M_{L} & M_{D}^{T}+\delta M_{D}^{T} \\
M_{D}+\delta M_{D} & \hat{M}_{R}+\delta M_{R}
\end{array}\right) \approx\left(\begin{array}{cc}
\delta M_{L} & M_{D}^{T} \\
M_{D} & \hat{M}_{R}
\end{array}\right),
$$

where the $0_{3 \times 3}$ matrix appearing at tree level (1.3) is replaced by the contribution $\delta M_{L}$. This correction a symmetric matrix, it dominates among all the sub-matrices of corrections. Neglecting the sub-dominant pieces in (1.7), one-loop corrections to the neutrino masses originate via the selfenergy function $\Sigma_{L}^{S}(0)=\Sigma_{L}^{S(Z)}(0)+\Sigma_{L}^{S\left(G^{0}\right)}(0)+\Sigma_{L}^{S\left(H^{0}\right)}(0)$, where the $\Sigma_{L}^{S\left(Z, G^{0}, H^{0}\right)}(0)$ functions arise from the self-energy Feynman diagrams and are evaluated at zero external momentum squared. In the calculation of the self energies the neutrino couplings to the $Z$ boson as well as the Higgs and Goldstone bosons are determined by eqs. (1.5) and (1.6). Each diagram contains a divergent piece but when summing up the three contributions the result turns out to be finite.

The final expression for one-loop corrections is given by [5]

$$
\begin{aligned}
\delta M_{L}= & \sum_{b} \frac{1}{32 \pi^{2}} \Delta_{b}^{T} U_{R}^{*} \hat{m}\left(\frac{\hat{m}^{2}}{m_{H_{b}^{0}}^{2}}-\mathbb{1}\right)^{-1} \ln \left(\frac{\hat{m}^{2}}{m_{H_{b}^{0}}^{2}}\right) U_{R}^{\dagger} \Delta_{b} \\
& +\frac{3 g^{2}}{64 \pi^{2} m_{W}^{2}} M_{D}^{T} U_{R}^{*} \hat{m}\left(\frac{\hat{m}^{2}}{m_{Z}^{2}}-\mathbb{1}\right)^{-1} \ln \left(\frac{\hat{m}^{2}}{m_{Z}^{2}}\right) U_{R}^{\dagger} M_{D}
\end{aligned}
$$

where $\operatorname{sum} \sum_{b}$ runs over all neutral physical Higgses $H_{b}^{01}$.

\section{Case $n_{R}=1$}

First we consider the minimal extension of the standard model adding only one right-handed field $v_{R}$ to the three left-handed fields contained in $v_{L}$.

We use the parametrization of $\Delta_{1}=\frac{\sqrt{2} m_{D}}{v} \vec{a}_{1}^{T}$ and $\Delta_{2}=\frac{\sqrt{2} m_{D}}{v} \vec{a}_{2}^{T}$ with $\left|\vec{a}_{1}\right|=1$ and $\left|\vec{a}_{2}\right|=1$. Diagonalization of the symmetric mass matrix $M_{V}$ (1.3) in block form is

$$
U^{T} M_{v} U=U^{T}\left(\begin{array}{cc}
0 & m_{D} \vec{a}_{1} \\
m_{D} \vec{a}_{1}^{T} & \hat{M}_{R}
\end{array}\right) U=\left(\begin{array}{cc}
\hat{M}_{l} & 0 \\
0 & \hat{M}_{h}
\end{array}\right) .
$$

The non zero masses in $\hat{M}_{l}$ and $\hat{M}_{h}$ are determined analytically by finding eigenvalues of the hermitian matrix $M_{v} M_{v}^{\dagger}$. These eigenvalues are the squares of the masses of the neutrinos $\hat{M}_{l}=$ $\operatorname{diag}\left(0,0, m_{l}\right)$ and $\hat{M}_{h}=m_{h}$. Solutions $m_{D}^{2}=m_{h} m_{l}$ and $m_{R}^{2}=\left(m_{h}-m_{l}\right)^{2} \approx m_{h}^{2}$ correspond to the seesaw mechanism.

\footnotetext{
${ }^{1}$ In our analysis we fix $m_{H_{1}^{0}}=125 \mathrm{GeV}$ but $m_{H_{2}^{0}}$ and $m_{H_{3}^{0}}$ we generate randomly in the range 1 to $1000 \mathrm{GeV}$.
} 
We can construct the diagonalization matrix $U$ for the tree level from two diagonal matrices of phases and three rotation matrices $U_{\text {tree }}=U_{\phi}\left(\phi_{i}\right) U_{12}\left(\alpha_{1}\right) U_{23}\left(\alpha_{2}\right) U_{34}(\beta) U_{i}$, where the angle $\beta$ is determined by the masses $m_{l}$ and $m_{h}$. The values of $\phi_{i}$ and $\alpha_{i}$ can be chosen to cover variations in $M_{D}$.

For calculation of radiative corrections we use following set of orthogonal complex vectors: $b_{Z}=(i, 0), b_{1}=(1,0), b_{2}=(0, i)$ and $b_{3}=(0,1)$. Diagonalization of the mass matrix after calculation of one-loop corrections is performed with a unitary matrix $U_{\text {loop }}=U_{\text {egv }} U_{\varphi}\left(\varphi_{1}, \varphi_{2}, \varphi_{3}\right)$, where $U_{\text {egv }}$ is an eigenmatrix of $M_{v}^{(1)} M_{v}^{(1) \dagger}$ and $U_{\varphi}$ is a phase matrix. The second light neutrino obtains its mass from radiative corrections. The third light neutrino remains massless.

It is possible to estimate masses of the light neutrinos from experimental data of solar and atmospheric neutrino oscillations [6] assuming that the lightest $m_{l_{3}}=0$. Considering the normal ordering of the light neutrinos we receive $m_{l_{1}}=5.0 \pm 0.2 \times 10^{-11} \mathrm{GeV}$ and $m_{l_{2}}=8.7 \pm 0.3 \times$ $10^{-12} \mathrm{GeV}$. Numerical analysis shows that we can reach those values for a heavy singlet with the mass bigger than $830 \mathrm{GeV}$, see Fig. 1 .

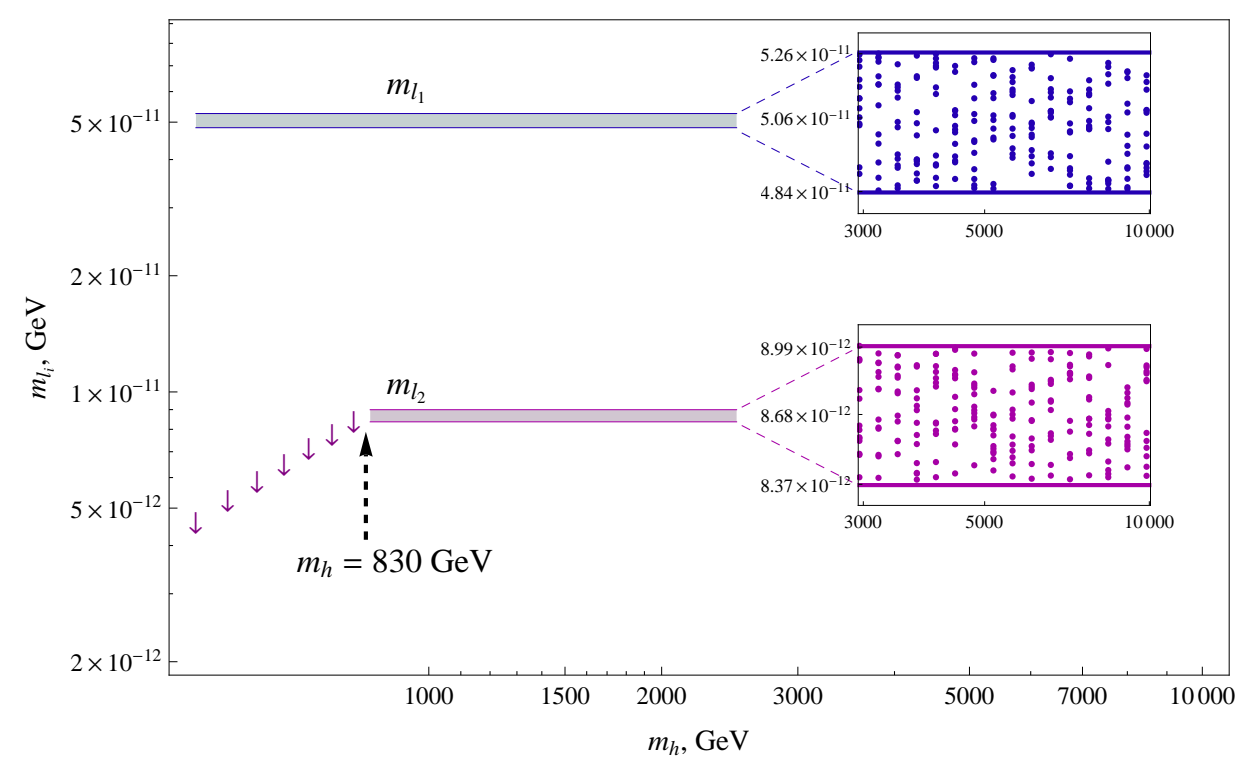

Figure 1: Calculated masses of two light neutrinos as a function of the heavy neutrino mass $m_{h}$. The mass of the third light neutrino is zero, when $n_{R}=1$. Solid lines show the boundaries of allowed neutrino mass ranges when the model parameters are constrained by the experimental data on neutrino oscillations. The purple arrows indicates the values of $m_{l_{2}}$ neutrino mass which don't satisfy allowed experimental neutrino mass ranges. Due to the scale, the band of the allowed $m_{l_{1}}$ and $m_{l_{2}}$ values are close to each other accordingly their values are shown separately in the right plots.

\section{Case $n_{R}=2$}

If we add two singlet fields $v_{R}$ to the three left-handed fields $v_{L}$, the radiative corrections give masses to all three light neutrinos. 
Now we parametrize $\Delta_{1}=\frac{\sqrt{2}}{v}\left(\begin{array}{c}m_{D_{2}} \vec{a}_{1}^{T} \\ m_{D_{1}} \vec{b}_{1}^{T}\end{array}\right)$ and $\Delta_{2}=\frac{\sqrt{2}}{v}\left(\begin{array}{c}m_{D_{2}} \vec{a}^{T} \\ m_{D_{1}} \vec{b}_{2}^{T}\end{array}\right)$ with $\left|\vec{a}_{1}\right|=1,\left|\overrightarrow{b_{1}}\right|=1$, $\left|\vec{a}_{2}\right|=1$ and $\left|\overrightarrow{b_{2}}\right|=1$. Diagonalizing the symmetric mass matrix $M_{v}$ (1.3) in block form we write:

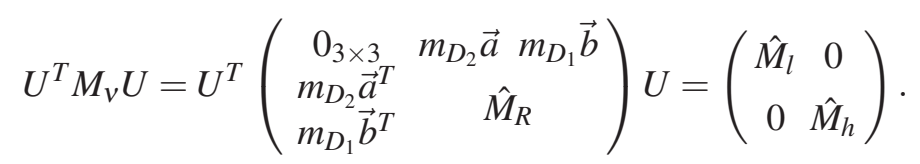

The non zero masses in $\hat{M}_{l}$ and $\hat{M}_{h}$ are determined by the seesaw mechanism: $m_{D_{i}}^{2} \approx m_{h_{i}} m_{l_{i}}$ and $m_{R_{i}}^{2} \approx m_{h_{i}}^{2}, i=1,2$. Here we use $m_{1}>m_{2}>m_{3}$ ordering of masses. The third light neutrino is massless at tree level.

The diagonalization matrix for tree level $U_{\text {tree }}=U_{12}\left(\alpha_{1}, \alpha_{2}\right) U_{\text {egv }}\left(\beta_{i}\right) U_{\phi}\left(\phi_{i}\right)$ is composed of a rotation matrix, an eigenmatrix of $U_{12}^{T} M_{v} M_{v}^{\dagger} U_{12}^{*}$ and a diagonal phase matrix, respectively.

For calculation of radiative corrections we use same set of orthogonal complex vectors $b_{i}$ as in first case. Diagonalization of the mass matrix including the one-loop correction is performed with a unitary matrix $U_{\text {loop }}=U_{\text {egv }} U_{\varphi}\left(\varphi_{i}\right)$, where $U_{\text {egv }}$ is the eigenmatrix of $M_{v}^{(1)} M_{v}^{(1) \dagger}$ and $U_{\varphi}$ is a phase matrix.

In numerical calculations the model parameters as well as the derived masses of the light neutrinos are obtained in several steps. First, the diagonal mass matrix for tree level is constructed. The lightest neutrino is massless, and the masses of other two light neutrinos are estimated from experimental data on solar and atmospheric neutrino oscillations. The masses of the heavy neutrinos are input parameters. This diagonal matrix is used to constrain the parameters $\alpha_{i}$ and $\phi_{i}$ that enter the tree-level mass matrix $M_{v}$ and its diagonalization matrix $U_{\text {tree }}$. Then the diagonalization matrix is used to evaluate one-loop corrections to the mass matrix. Diagonalization of the corrected mass matrix yields masses for three light neutrinos. If the calculated mass difference is compatible with the experimental neutrino mass difference, the parameter set is kept. Otherwise, another set of the parameters is generated. Figure 2 illustrate the obtained results. Both normal and inverted neutrino mass orderings are considered.
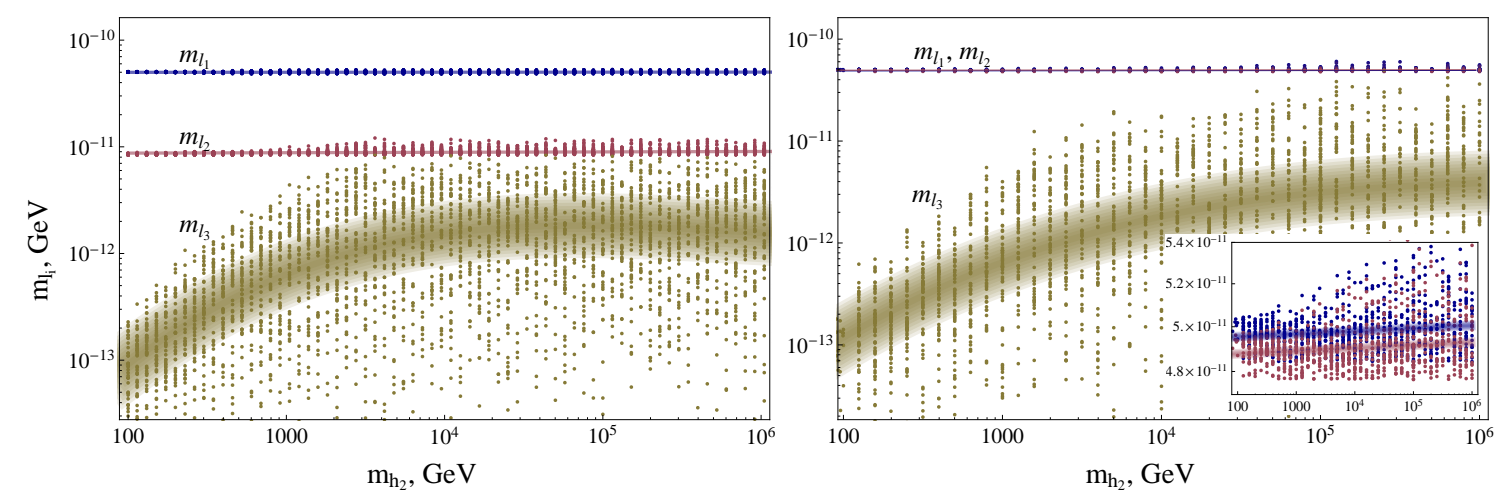

Figure 2: The masses $m_{l_{i}}$ of the light neutrinos as functions of the heaviest right-handed neutrino mass $m_{h_{1}}$, for the case $n_{R}=2$. The value of second heaviest right-handed neutrino mass is fixed at $m_{h_{2}}=100 \mathrm{GeV}$. Plot in the left represent normal hierarchy than the plot in the right represent inverted hierarchy of the light neutrinos. The wide solid lines indicate the place of the most frequent values of the scatter data. In inverted hierarchy case the nearly degenerate masses $m_{l_{1}}$ and $m_{l_{2}}$ are shown separately in the lower right plot. 


\section{Conclusions}

For the case $n_{R}=1$ we can match the differences of the calculated light neutrino masses to $\Delta m_{\odot}^{2}$ and $\Delta m_{\mathrm{atm}}^{2}$ with the mass of a heavy singlet bigger than $830 \mathrm{GeV}$. Only normal ordering of neutrino masses is possible.

In the case $n_{R}=2$ we obtain three non vanishing masses of light neutrinos for normal and inverted hierarchies. The numerical analysis shows that the values of light neutrino masses (especially of the lightest mass) depend on the choice of the heavy neutrinos masses. The radiative corrections generate the lightest neutrino mass and have a big impact on the second lightest neutrino mass.

In future we plan to apply our parametrization to study the $\tau$ polarization coming from the decay of a $W$ boson in the data of the CMS experiment at LHC and thus determine restrictions to the parameters of the neutrino sector.

Acknowledgements. The authors thank Luis Lavoura for valuable discussions and suggestions. This work was supported by European Union Structural Funds project "Postdoctoral Fellowship Implementation in Lithuania".

\section{References}

[1] M. Gell-Mann, P. Ramond, and R. Slansky, in Supergravity, Proceedings of the Workshop, Stony Brook, New York, 1979, edited by F. van Nieuwenhuizen and D. Freedman (North Holland, Amsterdam, 1979).

[2] J. Schechter and J. W. F. Valle, Phys. Rev. D 22 (1980) 2227.

[3] W. Grimus and H. Neufeld, Nucl. Phys. B 325 (1989) 18.

[4] W. Grimus and L. Lavoura, Soft lepton-flavor violation in a multi-Higgs-doublet seesaw model, Phys. Rev. D 66 (2002) 014016 [hep-ph/ 0204070$].$

[5] W. Grimus and L. Lavoura, One-loop corrections to the seesaw mechanism in the multi-Higgs-doublet Standard Model, Phys. Lett. B 546 (2002) 86 [hep-ph/ 0207229 ].

[6] M. C. Gonzalez-Garcia, M. Maltoni, J. Salvado and T. Schwetz, Global fit to three neutrino mixing: critical look at present precision, arXiv:1209.3023. 